\title{
Static and Dynamic Characteristics of Cerebral Blood Flow during the Resting State
}

\author{
Qihong Zou ${ }^{1,2}$, Changwei W. Wu ${ }^{1,3}$, Elliot A. Stein ${ }^{1}$, Yufeng Zang ${ }^{2}$, and Yihong Yang ${ }^{1}$ \\ ${ }^{1}$ Neuroimaging Research Branch, National Institute on Drug Abuse, National Institutes of Health, \\ Baltimore, MD 21224, USA \\ ${ }^{2}$ State Key Laboratory of Cognitive Neuroscience and Learning, Beijing Normal University, China \\ ${ }^{3}$ Department of Electrical Engineering, National Taiwan University, Taipei, Taiwan
}

\begin{abstract}
In this study, the static and dynamic characteristics of cerebral blood flow (CBF) in the resting state were investigated using an arterial spin labeling (ASL) perfusion imaging technique. Consistent with previous PET results, static CBF measured by ASL was significantly higher in the posterior cingulate cortex (PCC), thalamus, insula/superior temporal gyrus (STG) and medial prefrontal cortex (MPFC) than the average $\mathrm{CBF}$ of the brain. The dynamic measurement of $\mathrm{CBF}$ fluctuations showed high correlation (functional connectivity) between components in the default mode network. These brain regions also had high local temporal synchrony and high fluctuation amplitude, as measured by regional homogeneity (ReHo) and amplitude of low-frequency fluctuations (ALFF) analyses. The spatial pattern of the static CBF correlated well with that of the dynamic indices. The high static and dynamic activity in the PCC, MPFC, insula/STG and thalamus suggest that these regions play a vital role in maintaining and facilitating fundamental brain functions.
\end{abstract}

\section{Keywords}

CBF; static; dynamic; functional connectivity; ALFF; ReHo; resting state

\section{Introduction}

There has been growing interest in resting brain activity (Raichle et al., 2001; Fox and Raichle 2007; Greicius 2008). Positron emission tomography (PET) first demonstrated that a set of brain regions including posterior cingulate cortex (PCC), medial prefrontal cortex (MPFC), thalamus and insula exhibit higher cerebral blood flow (CBF) than the whole brain average in the resting state (Raichle et al., 2001). CBF in the majority of these regions decreases from its baseline level during a wide range of goal-directed tasks (Shulman et al., 1997; Mazoyer et al., 2001). Together, these brain regions have been called the default mode network (DMN). In contrast to the observation of static CBF in PET studies, dynamic interactions between brain

\footnotetext{
Please send correspondences to: Yihong Yang, Ph.D. Neuroimaging Research Branch, National Institute on Drug Abuse, NIH 251 Bayview Blvd., Suite 200, Baltimore, MD 21224, USA E-mail: yihongyang@ intra.nida.nih.gov Tel: 443-740-2648 Fax: 443-740-2816. Yufeng Zang, M.D. State Key Laboratory of Cognitive Neuroscience and Learning, Beijing Normal University No. 19 Xinjiekouwai Street, Beijing 100875, China. Email: zangyf@ bnu.edu.cn Tel: 86-10-58801023 Fax: 86-10-58801023.

Publisher's Disclaimer: This is a PDF file of an unedited manuscript that has been accepted for publication. As a service to our customers we are providing this early version of the manuscript. The manuscript will undergo copyediting, typesetting, and review of the resulting proof before it is published in its final citable form. Please note that during the production process errors may be discovered which could affect the content, and all legal disclaimers that apply to the journal pertain.
} 
regions have been revealed using resting-state functional magnetic resonance imaging (fMRI) (Biswal et al., 1995). Most of these fMRI studies utilized the synchrony of spontaneous fluctuations in the blood oxygenation level dependent (BOLD) signal to identify coherent brain networks and to assess alterations in connectivity strength in various brain disorders (e.g., Greicius et al., 2004; Bluhm et al., 2007; Greicius 2008; Hong et al., 2009). While the dynamic signals in the DMN are highly correlated (Greicius et al., 2003; Fransson 2005; Fox et al., 2005; Beckmann et al., 2005), the static and dynamic characteristics of resting-state signals have not been systematically studied under a single modality within the same subjects.

Perfusion imaging based on arterial spin labeling (ASL) has been widely used to measure resting-state blood flow in the brain (Detre et al., 1992). ASL approaches can also be utilized to measure dynamic, spontaneous CBF changes in the resting state. An early study demonstrated that spontaneous low frequency $(<0.1 \mathrm{~Hz})$ flow-weighted fluctuations are highly synchronized within the motor system (Biswal et al., 1997b). De Luca et al. (2006) observed several brain networks using resting-state ASL data from a single subject. Recently, Chuang et al. (2008) developed a strategy to reduce BOLD contamination in the resting-state CBF fluctuations and reported connectivity within the sensorimotor network.

Using ASL, we investigated the spatial distribution of static and dynamic CBF in the resting state within the same subjects. A multi-slice, pulsed ASL (PASL) technique was utilized to collect CBF time courses from healthy subjects. Static CBF was measured by voxel-wise averaging the CBF values over the time domain. Dynamic characteristics of the brain were assessed using three methods. Classic cross-correlation analysis with a predefined "seed" was used to examine functional connectivity to the seed. We also assessed the characteristics of the CBF dynamic fluctuations from the aspect of local information, which might provide novel and complementary information to the correlation analysis. Amplitude of low-frequency fluctuations (ALFF) (Zang et al., 2007), which quantifies the strength of the fluctuations in each voxel, was adopted in the current study to depict the local intensity of CBF fluctuations. Regional homogeneity (ReHo) (Zang et al., 2004), which reflects local synchrony by calculating similarity of dynamic fluctuations of voxels within a given cluster, was also used in the analysis to reveal local synchrony of CBF fluctuations. Since CBF is a single physiological parameter (vs. BOLD which is a composite of several parameters) and is probably more closely related to cerebral metabolism than BOLD, ReHo and ALFF results from $\mathrm{CBF}$ data may be more physiologically relevant than those from BOLD.

\section{Materials and Methods}

\section{Participants}

Twelve healthy subjects ( $26.3 \pm 6.4$ years old, 9 females, and 3 males) participated in the study. All subjects were screened with a questionnaire to ensure no history of neurological illness, psychiatric disorders or past drug abuse. They were recruited under a protocol approved by the Institutional Review Board of the Intramural Research Program of the National Institute on Drug Abuse. Signed informed consent was obtained from all participants prior to study enrollment.

\section{Data acquisition}

Functional MRI data were collected on a 3T Siemens Allegra MR scanner (Siemens, Erlangen, Germany) equipped with a quadrature volume head coil. A PASL sequence based on the flowsensitive alternating inversion recovery (FAIR) echo-planar imaging (EPI) method was adopted for functional scans. Arterial blood was labeled with alternating slice-selective inversion recovery (label, SIR) and non-slice-selective inversion recovery (control, NSIR) scans. Important components of QUIPSS II (Wong et al., 1998), such as additional saturation 
pulses to control the time duration of the tagged bolus, were added into the sequence. These components make the PASL technique relatively insensitive to transit delays and improve the quantification of perfusion. Imaging parameters of the PASL sequence were as follows: TE $\left(\right.$ echo time) $/$ TR $\left(\right.$ repetition time) $/$ TI (inversion time) $=28 / 2000 / 1400 \mathrm{~ms}$, flip angle $=90^{\circ}$, bandwidth $=4112 \mathrm{~Hz} /$ pixel. The saturation pulse to control the tagged bolus was applied at $\mathrm{TI}_{1}=700 \mathrm{~ms}$, with a saturation thickness of $100 \mathrm{~mm}$ and a gap of $12 \mathrm{~mm}$ to the imaging slab. Ten oblique imaging slices (thickness $/ \mathrm{gap}=6 / 0 \mathrm{~mm}$, field of view $=220 \times 220 \mathrm{~mm}^{2}$, in-plane resolution $=3.44 \times 3.44 \mathrm{~mm}^{2}$ ) were prescribed to cover a large part of the default mode network (Raichel et al., 2001). The slab thickness for the SIR scans was approximately 1.4 times the imaging slab ( $84 \mathrm{~mm}$ thickness in total) to avoid artifacts in perfusion images due to imperfect transition of the slab profile (Yang et al., 1998). Head movement was minimized by using a polyurethane foam helmet individually made for each participant. Before the resting-state scan, subjects were instructed to rest with their eyes closed, not to think of anything in particular, and not to fall asleep during the acquisition period. Twelve minutes of continuous ASL data were acquired for each subject, corresponding to 360 measurements.

After the functional scans, a total of 19 NSIR-EPI images were acquired with multiple TIs (30, $80,130,180,230,330,430,530,630,730,830,1030,1230,1530,1830,2230,2730,3230$ and $3830 \mathrm{~ms}$ ) to generate $\mathrm{T}_{1}$ maps for image segmentation. For registration purposes, a set of high-resolution anatomical images were acquired using a 3-D magnetization prepared rapid gradient echo (MPRAGE) $\mathrm{T}_{1}$-weighted sequence $\left(256 \times 192 \times 160\right.$ matrix size; $1 \times 1 \times 1 \mathrm{~mm}^{3}$ inplane resolution; TI/TR/TE $=1000 / 2500 / 4.38 \mathrm{~ms}$; flip angle $=8^{\circ}$ ) on each subject.

\section{Data preprocessing}

A $\mathrm{T}_{1}$ map was curve-fitted for each subject using the NSIR-EPI images with multiple TIs, which was then used for structural segmentation into maps of gray matter, white matter, and cerebrospinal fluid (CSF) using a custom linear decomposition algorithm in MATLAB (MathWorks, Inc., CA).

The resting-state PASL data were preprocessed using the Analysis of Functional Neuroimaging (AFNI) software package (Cox, 1996), including the following: (1) slice time correction for acquisition time differences between slices; (2) head motion correction for head movement during the scan; (3) linear detrending of signal drift; (4) spatial normalization to standard Talairach and Tournoux (TT) space with a resampled resolution of $3 \times 3 \times 3 \mathrm{~mm}^{3}$ to facilitate group analysis; and (5) spatial smoothing with a 6-mm Gaussian kernel to minimize individual variance and enhance the signal-to-noise ratio (SNR). Potential contamination from BOLD was removed using the method proposed by Chuang et al. (2008). Each PASL dataset was

high-pass filtered at the cutoff frequency of $0.125 \mathrm{~Hz}$ (corresponding to $\frac{1}{4 T R}$ ) using Chebyshev type II filters in MATLAB. The PASL signal was demodulated to low frequency by multiplying $\cos (\pi n)(n$ is the scan number). Note that high-pass filtering followed by demodulation is the same as demodulation followed by low-pass filtering, and this process will produce similar results to sinc interpolation of the ASL time course to create time-matched control and label images followed by subtraction (Aguirre et al., 2002; Liu and Wong, 2005; Chuang et al., 2008). Potential contamination from physiological noise was reduced using independent component analysis (ICA) at a group level (Beckmann and Smith 2004), provided in the FMRIB Software Library (FSL; http://www.fmrib.ox.ac.uk/fsl/melodic/index.html). The resting-state PASL data of 12 subjects were automatically decomposed into 20 components. High spatial correlation of the components with CSF and/or vascular maps suggested the components to be artifact instead of representing neuronal activity (Stevens et al., in press). One component that had the highest average $z$-score in the vessel mask (http://www.bic.mni.mcgill.ca/brainweb/anatomic_normal_20.html) and another component that had the highest average $\mathrm{z}$-score in the cerebrospinal fluid (CSF) mask (prior probability 
template provided by FSL, and thresholded at 0.5 ) were considered irrelevant to neuronal function (Fig. 1). The majority of the voxels in the two components are located in the respective masks, although a small portion of the voxels (especially the CSF component) does not seem to overlap with the masks, probably due to imperfections of the ICA source separation. These two components were regressed out from the data as follows: a subject specific time course of each component was created by regressing the group component maps against the individual data (using fsl_glm implemented in FSL). Then the time courses corresponding to these two noise components were filtered out of each subject's data (using fsl_regfilt implemented in FSL).

\section{Data analysis}

Average CBF map (static CBF map) was calculated for each subject from the ICA denoised data. Similar to PET analyses (Raichle et al., 2001), the static CBF map of each subject was divided by the mean CBF values of that subject in a brain mask that was the intersection of scanned brain regions in all subjects. To reveal brain regions with CBF significantly higher than the global mean CBF, a voxel-wise one-sided one-sample $t$-test against 1 was performed on the normalized CBF maps (Raichle et al., 2001).

To evaluate the dynamic characteristics of $\mathrm{CBF}$, three approaches were used to analyze the PASL data after ICA denoising:

1. Seed-based correlation analysis was employed to examine the temporal relationship between the PCC and other brain regions. A 6-mm-diameter sphere centered at the Talairach coordinates of $[10,-54,14]$ (Wu C.W. et al., 2009) was defined as a seed region. A cross-correlation coefficient (CC) map was obtained by correlating the average time course of the seed region with each voxel's time course over the brain. Several nuisance covariates (six head motion parameters and average time course retrieved from the segmented white matter mask) were regressed out during the calculation of the CC map. The white matter signal was used as a nuisance covariate as it is assumed to not significantly reflect neuronal activity. Correlation coefficients were converted to $z$-scores by using Fisher's $r$-to- $z$ transformation to improve normality. To reveal the PCC correlation pattern, one-sample $t$-test was performed on individual $z$ maps to determine whether the $z$-scores significantly differed from zero.

2. Regional Homogeneity (ReHo) (Zang et al., 2004) was used to measure the similarity of time courses of the voxels within a given cluster (e.g., 27 nearest neighboring voxels). A custom software routine, Resting-State fMRI Data Analysis Toolkit (REST, http://restingfmri.sourceforge.net) was used for ReHo analysis in a voxelwise fashion. ReHo assumes that the time courses within a functional cluster are similar to each other. Kendall's coefficient concordance (KCC, ranged from 0 to 1 ) (Kendall and Gibbons 1990) was used to estimate the degree of similarity of multiple time courses (Zang et al., 2004), calculated as follows:

$$
W=\frac{\sum(R i)^{2}-n(\bar{R})^{2}}{\frac{1}{12} K^{2}\left(n^{3}-n\right)},
$$

where $W$ is the KCC value; $R i$ is the sum rank of the $i$ th time point;

$\bar{R}=((n+1) \quad K) \quad / \quad 2$ is the mean of the $R i$ 's; $K$ is the number of time courses within a measured cluster ( $K=27$ in the current study); $n$ is the number of the ranks ( $n=360$ in the current study). Similar to the static CBF map, the ReHo map of each subject 
was divided by that subject's mean ReHo value, and one-sided one-sample $t$-test against 1 were performed on the normalized maps.

3. Amplitude of low frequency fluctuations (ALFF) (Zang et al., 2007) was utilized to detect the strength of spontaneous fluctuations of the PASL signal in each voxel. Using REST, the time course of each voxel was transformed to the frequency domain, ALFF was calculated as the amplitude integral over a frequency range of $0-0.125 \mathrm{~Hz}$. Similar to the static CBF map and ReHo map, the ALFF map of each subject was divided by that subject's mean ALFF value, then one-sided one-sample $t$-test against 1 was performed on the normalized maps.

The effects of reduced degrees of freedom in the CBF time course due to signal processing (such as the Chebyshev filtering process) were taken into account in the $t$-tests, similar to that in a previous study (Fox et al., 2006). To control for Type I error in the resultant statistical maps, Monte Carlo simulations were performed using the AFNI AlphaSim program (parameters: individual voxel $p<5 \times 10^{-4}, 10,000$ simulations, FWHM $=6 \mathrm{~mm}$, within the brain mask). By iterating the process of random image generation, spatial correlation calculation, thresholding and clusters identification, an estimate of the overall significance level is achieved by various combinations of individual voxel probability and cluster size thresholds (Poline et al., 1997). A corrected significance level of $p<0.001$ for the resultant statistical maps was obtained by combining individual voxel $p<5 \times 10^{-4}$ with a minimum cluster size of $648 \mathrm{~mm}^{3}$. Based on the results of one-sample one-sided $t$-tests, four ROIs for the PCC, MPFC, insula/superior temporal gyrus (STG) and thalamus were defined to show the average static $\mathrm{CBF}, \mathrm{CBF}$ time courses, ReHo, and ALFF values within each ROI.

Finally, spatial correlations were calculated to measure spatial similarity between the statistical $t$ maps of static CBF and indices of dynamic CBF fluctuations. The spatial correlation was computed as

$$
R^{X, Y}=\frac{\sum_{i=1}^{N}\left(t_{i}^{X}-t_{i}^{X}\right)\left(t_{i}^{Y}-\overline{t_{i}^{Y}}\right)}{\sqrt{\sum_{i=1}^{N}\left(t_{i}^{X}-\bar{t}_{i}^{X}\right)^{2}\left(t_{i}^{Y}-\bar{t}_{i}^{Y}\right)^{2}}},
$$

where $N$ is the number of voxels in the intersection mask of scanned regions in all subjects, $t_{i}^{X}$ is the $t$ value from static CBF map at voxel $i$, and $t_{i}^{Y}$ is the $t$ value from dynamic CBF map (PCC-based correlation, ReHo or ALFF) at voxel $i$.

For each of the four statistical $t$ maps, the significant voxels (uncorrected $p<2.5 \times 10^{-4}$ ) were labeled as 1 , and non-significant voxels were labeled as 0 . Combinations of two of the static CBF, ALFF and ReHo maps were added together (Fig. 6A, B and C), and all four binary maps were added together (Fig. 6D) to show the overlap of these $t$ maps (with minimum cluster size of $540 \mathrm{~mm}^{3}$ ).

\section{Results}

\section{Static characteristics of CBF in the resting brain}

Static CBF $t$ map is shown in Fig. 2A. Consistent with a previous PET study (Raichle et al., 2001), static CBF in the PCC, MPFC, thalamus and insula/STG were significantly higher than whole brain mean CBF. Fig. 2B shows that the average static CBF within these four ROIs was significantly higher than the global average (which was normalized as 1). 


\section{Dynamic characteristics of $\mathrm{CBF}$ in the resting brain}

Dynamic CBF fluctuations of the PCC were significantly correlated with brain regions in the DMN, such as MPFC, inferior parietal lobule and insula/STG (Fig. 3A). The average CBF time courses within the PCC and MPFC ROIs from a representative participant are shown in Fig. 3B. The correlation coefficient between these two ROIs in this subject was 0.53.

The brain regions that showed higher local synchrony (i.e., higher ReHo) are shown in Fig. 4A. The PCC, MPFC, insula/STG and thalamus had higher within-region local synchrony than other brain regions (Fig. 4). The ALFF map (Fig. 5) shows a very similar pattern with the ReHo and static CBF maps.

The spatial correlation coefficients between $t$ maps of static CBF and indices of dynamic PASL data (PCC-based correlation, ReHo and ALFF) were 0.40, 0.63 and $0.72(p<0.05)$, respectively. Fig. 6 shows the overlap of brain regions obtained from the static and dynamic indices. The overlapped brain regions were primarily in the PCC, MPFC, insula/STG and thalamus.

\section{Discussion}

In the current study, we investigated the static and dynamic characteristics of resting-state $\mathrm{CBF}$ signals recorded using a PASL technique. Static CBF was significantly higher in the PCC, thalamus, insula/STG and MPFC than the whole brain average CBF value. The dynamic measurement of CBF fluctuations showed that the PCC was highly synchronized with brain regions primarily in the DMN (Raichle et al., 2001). The dynamic CBF fluctuations of these four regions also showed high local synchrony (ReHo) and fluctuation amplitude (ALFF).

Compared to PET and BOLD, ASL has the abilities to study both the static and dynamic CBF characteristics in a single session within the same subjects. Static CBF measurements by ASL imaging have been applied in clinical settings, including cerebrovascular disease (Detre et al., 1998), brain tumors (Gaa et al., 1996), aging and neurodegenerative disorders (Johnson et al., 2005), and pediatric diseases (Strouse et al., 2006). Dynamic changes of CBF measured by ASL can be used to study resting-state brain function (Biswal et al., 1997b; De Luca et al., 2006; Chuang et al., 2008; Wu C.W. et al., 2009), similar to the more commonly used BOLD techniques. In this study, ASL was used to simultaneously assess the static and dynamic CBF properties in the DMN within the same subject group, which should reduce variations between subject groups and between imaging modalities.

We employed high-pass filtering (Chuang et al., 2008) and independent component analysis (ICA) denoising (Beckmann and Smith 2004) to reduce potential BOLD contamination and physiological aliasing, respectively. Chuang et al. (2008) proposed a method to suppress BOLD contributions using the aliased high frequency range CBF signal. The feasibility of this method was demonstrated by their studies in the bilateral sensorimotor cortices. In the current study, we adopted this method to reduce the potential contamination from BOLD in the CBF signals. The relatively low temporal resolution of ASL imaging makes it sensitive to physiological signals irrelevant to neural functions. ICA is a fully data-driven method that is able to decompose the resting-state fMRI signal into multiple spatially distributed signal and noise components. We used group ICA (Beckmann and Smith 2004) to suppress potential physiological noise from the CBF signal caused by its low sampling rate. These processing steps are necessary to ensure the successful utilization of ASL signal for resting-state analyses.

Static characteristics of CBF signals were studied in the current study. Despite being widely used in research and clinical applications, ASL has not been used, to our knowledge, in the assessment of spatial distribution of static CBF when compared to the whole brain average. 
Using PASL, the current study demonstrated that static CBF in the PCC, thalamus, insula/STG and MPFC were significantly higher than the global average (Fig. 2). Our findings agree well with a previous PET Study. Using $\mathrm{O}^{15}$ PET, Raichle et al. (2001) found that CBF in the PCC and MPFC were significantly higher than its global mean in three groups of subjects. The consistency between the ASL measurements in our study and the previous PET measurements indicates the potential to quantify static CBF without radiation exposure (Ye et al., 2000).

We also investigated the dynamic characteristics of CBF signals from three different aspects: remote synchrony (functional connectivity), local synchrony (Reho) and local activity amplitude (ALFF). Almost all the previous CBF-based resting-state studies used functional connectivity analyses (Biswal et al., 1997b; De Luca et al., 2006; Chuang et al., 2008). Using the PCC as a seed, our correlation analysis demonstrated that dynamic CBF changes of the PCC were significantly correlated with those of brain regions in the DMN, including the MPFC, inferior parietal lobe and insula/STG (Fig. 3). These results were similar to those seen in previous BOLD-based (Greicius et al., 2003; Fox et al., 2005) and perfusion-based (Wu C.W. et al., 2009) connectivity studies. Some BOLD-based functional connectivity studies (e.g. Fox et al., 2005) showed negative correlations between the PCC and insula/STG. One explanation for this inconsistency could be whether the global mean signals were regressed out (Murphy et al., 2009; Fox et al., 2009) before functional connectivity analysis. In the current study, only the mean signals in the white matter mask were used to regress out fluctuations unlikely to be relevant to neuronal activity (Hong et al., 2009). However, global signal removal is still somewhat controversial and needs further comprehensive study. It should be noted that functional connectivity indicates remote synchrony between two regions, which does not reflect the activity of one region. ReHo and ALFF were thus employed to further explore the local dynamics of the CBF signals. ReHo (Zang et al., 2004) has been used to depict local temporal synchrony, while ALFF (Zang et al., 2007; Biswal et al., 1995; Kiviniemi et al., 2000 ) is used as a measurement of local activity amplitude.

Previous studies have shown that a decrease of mean arterial pressure (MAP) increased the amplitude of CBF and BOLD fluctuations (Hudetz et al., 1992; Kannurpatti et al., 2008). In the present study, high ALFF of CBF fluctuations are shown in the PCC, MPFC, insula/STG and thalamus, together with high static CBF in some of these regions (Fig. 2 and Fig. 5). Although the exact reason for the discrepancy between our results and previous findings is not known, our results seem to support the hypothesis that the CBF (and BOLD) fluctuations during normal physiological conditions in the abovementioned areas are dominantly neuronally driven rather than of vasomotor origin, since low CBF with high ALFF would suggest vasomotor fluctuation dominance. Of course, further studies are needed to test this hypothesis.

While ALFF measures the amplitude of dynamic spontaneous activity fluctuation within a single voxel, ReHo measures the local synchrony of spontaneous activity within a cluster of neighboring voxels (Zang et al., 2004). In neurophysiological studies, single unit activity (SUA) reflects the action potentials of a single neuron (or sometimes a few neighboring neurons), while the local field potential (LFP) reflects the integration and summation of the input and local processing of neural activity within a couple of millimeters from the recording electrode (Logothetis et al., 2001). From a more macroscopic view of hemodynamics, ReHo may provide an integrative measure of a functional cluster. In this study, although most regions in the DMN showed both higher ALFF and ReHo, discrepancies still exist in some regions, (e.g., the thalamus, Fig. 6C), suggesting that the ALFF and ReHo may reflect, at least to some extent, different aspects of spontaneous brain activity. In fact, ReHo has been used as a potentially useful marker of abnormal resting brain synchronous activity in various brain disorders, such as ADHD (Cao et al., 2006; Zhu et al., 2008), schizophrenia (Liu et al., 2006), aging (Wu et al., 2007), AD (He et al., 2007) and Parkinson's Disease (Wu T. et al., 2009). 
The spatial distribution of the static $\mathrm{CBF}$ and the indices of dynamic $\mathrm{CBF}$ fluctuations were very similar (Figs. 2A, 3A, 4A and 5A). The PCC, MPFC, insula/STG and thalamus consistently showed high static CBF, strong cross-correlation, ReHo and ALFF (Fig. 6). Our results indicate that these brain regions presumably constitute a unified system in the brain, characterized as having a high baseline blood flow (metabolism), high synchrony between network components, high local synchrony and local fluctuation amplitude. The significantly higher static and dynamic activity (compared to the global average) suggests that these regions might play a critical role in communication and coordination in the brain.

From the prospect of energy consumption, the human brain is a large energy consuming organ that represents about $2 \%$ of the body weight, while accounting for $20 \%$ of the total body energy consumption. Furthermore, much of the brain's energy consumption is not attributed to external stimuli but to intrinsic activity (Raichle 2006). Thus, these highest energy consuming brain regions, i.e., the PCC, MPFC, insula/STG and thalamus, in the highest energy consuming organ of the human body, appear to be essential components in maintaining and facilitating resting state brain functions. Based on network analysis of the resting-state BOLD signal, the PCC, STG, thalamus and anterior cingulate have been demonstrated to act as functional core regions (Achard et al., 2006; van den Heuvel et al., 2008). Furthermore, using DTI-based tractography the PCC was demonstrated to be a hub region that widely connects with other regions (Gong et al., 2009). Using diffusion spectrum imaging, Hagmann et al. (2008) showed that the PCC and insula/STG constituted a structural core of large-scale structural brain networks that share a high degree of anatomical connectivity. Similarly, the PCC and insula show a critical and central role in anatomical networks estimated from diffusion-weighted MRI (Iturria-Medina et al., 2008). These structural connectivity studies point to similar brain regions as revealed from resting-state functional connectivity studies. It would thus be reasonable to predict that any damage to these key regions, such as in Alzheimer's disease, might severely impact brain function (Greicius et al., 2004).

It should be noted that a region with high static CBF might tend to have high ALFF, if the CBF fluctuation level is proportional to baseline $\mathrm{CBF}$. Our data show overlaps of high static CBF and ALFF in some regions of the PCC, MPFC, thalamus and insula/STG (Fig.6A). However, a region with high baseline CBF doesn't always show high fluctuation amplitude. For instance, the dorsal part of MPFC shows high ALFF, but its static CBF is not significantly higher than the global average (Fig. 6A). On the other hand, the striatum illustrates high static CBF, while its ALFF does not (Fig. 6A). These data suggest that the high ALFF in this study can not be attributed solely to high static CBF. Similarly, high static CBF might also give rise to high ReHo due to high SNR. Recently, Liau et al. (2008) demonstrated that the SNR of functional $\mathrm{CBF}$ response is proportional to its baseline CBF. In the present study, the high static $\mathrm{CBF}$ in the PCC, MPFC, thalamus and insula/STG might contribute to the high ReHo in these regions. However, regions with high static CBF, such as the striatum, may not necessarily have high ReHo (Fig. 6B). Furthermore, regions with high ReHo are not always accompanied by high static CBF, such as the dorsal part of the PCC (Fig. 6B). Since ReHo calculates crosscorrelation between local voxels, the temporal synchronization of the time courses between these voxels is likely to dominate the ReHo calculation, with SNR being a secondary factor. In addition, in BOLD-based resting-state studies, although the intensity of the BOLD signal in the DMN is lower than that in the CSF, ALFF and ReHo of BOLD signals have been shown to be significantly higher in the DMN that those in the CSF (Zang et al., 2007; Long et al., 2008; Zou et al., 2008). Together, these findings suggest that higher ALFF and ReHo in the DMN are not simply due to the high CBF baseline or SNR, but rather reflect the amplitude of local fluctuations and local synchrony.

It should also be noted that high-pass filtering followed by demodulation may not remove multiplicative BOLD contamination in the entire frequency band. The high-pass filtering on 
the CBF time courses was to "minimize" the BOLD contamination by removing low-frequency BOLD signals $(<0.125 \mathrm{~Hz})$, the main component of resting-state fluctuations. Recent studies showed that BOLD fluctuations in the high-frequency band $(>0.125 \mathrm{~Hz})$ were low in the gray matter, while relatively high in the CSF and white matter (Zou et al., 2008; Zuo et al., 2009). In addition, the high-frequency BOLD fluctuations were not correlated within specific functional networks (Chuang et al., 2008; Wu et al., 2008). The results in the present study showed more fluctuations in the gray matter than in the CSF and white matter and the observed fluctuations were significantly correlated within the default mode network, suggesting that the low-frequency CBF-weighted fluctuations dominated in our observations. Quantitatively, the spectral power of BOLD signal from the PCC in the high-frequency range $(0.125-0.25 \mathrm{~Hz})$ was approximately $17 \%$ of that in the whole frequency range $(0-0.25 \mathrm{~Hz})$ (Zou et al., 2008). Thus, the detected functional connectivity, ReHo and ALFF in the present study were mainly from CBF-weighted signal, rather than multiplicative BOLD contamination.

In summary, we investigated both the static and dynamic characteristics of resting human brain activity using a PASL technique. The relatively high temporal resolution of PASL makes it possible to simultaneously assess the static and dynamic CBF properties by the same imaging modality within the same subjects. Our data show that static CBF was significantly higher in PCC, thalamus, insula/STG and MPFC than the global brain blood flow average, consistent with previous PET observations. Dynamic analyses showed that these brain regions are highly correlated with the PCC, and possess high local synchrony and high fluctuation amplitude. The spatial distributions of the static and dynamic indices are well correlated. Together, our results support the importance of these DMN brain regions in maintaining and facilitating basic brain functions at rest. These results may also suggest that any damage to these brain regions might severely impact normal brain functions.

\section{ACKNOWLEDGEMENTS}

This work was supported by the Intramural Research Program of the National Institute on Drug Abuse (NIDA), National Institute of Health (NIH).

\section{References}

Achard S, Salvador R, Whitcher B, Suckling J, Bullmore E. A resilient, low-frequency, small-world human brain functional network with highly connected association cortical hubs. J.Neurosci 2006;26:63-72. [PubMed: 16399673]

Aguirre GK, Detre JA, Zarahn E, Alsop DC. Experimental design and the relative sensitivity of BOLD and perfusion fMRI. Neuroimage 2002;15:488-500. [PubMed: 11848692]

Beckmann CF, DeLuca M, Devlin JT, Smith SM. Investigations into resting-state connectivity using independent component analysis. Philosophical Transactions of the Royal Society B-Biological Sciences 2005;360:1001-1013.

Beckmann CF, Smith SM. Probabilistic independent component analysis for functional magnetic resonance imaging. IEEE Trans.Med.Imaging 2004;23:137-152. [PubMed: 14964560]

Biswal B, Hudetz AG, Yetkin FZ, Haughton VM, Hyde JS. Hypercapnia reversibly suppresses lowfrequency fluctuations in the human motor cortex during rest using echo-planar MRI. J.Cereb.Blood Flow Metab 1997a;17:301-308. [PubMed: 9119903]

Biswal B, Yetkin FZ, Haughton VM, Hyde JS. Functional connectivity in the motor cortex of resting human brain using echo-planar MRI. Magn Reson.Med 1995;34:537-541. [PubMed: 8524021]

Biswal BB, Van Kylen J, Hyde JS. Simultaneous assessment of flow and BOLD signals in resting-state functional connectivity maps. NMR Biomed 1997b;10:165-170. [PubMed: 9430343]

Bluhm RL, Miller J, Lanius RA, Osuch EA, Boksman K, Neufeld RW, Theberge J, Schaefer B, Williamson P. Spontaneous low-frequency fluctuations in the BOLD signal in schizophrenic patients: anomalies in the default network. Schizophr.Bull 2007;33:1004-1012. [PubMed: 17556752] 
Cao Q, Zang Y, Sun L, Sui M, Long X, Zou Q, Wang Y. Abnormal neural activity in children with attention deficit hyperactivity disorder: a resting-state functional magnetic resonance imaging study. Neuroreport 2006;17:1033-1036. [PubMed: 16791098]

Chuang KH, van Gelderen P, Merkle H, Bodurka J, Ikonomidou VN, Koretsky AP, Duyn JH, Talagala SL. Mapping resting-state functional connectivity using perfusion MRI. Neuroimage 2008;40:15951605. [PubMed: 18314354]

Cox RW. AFNI: software for analysis and visualization of functional magnetic resonance neuroimages. Comput.Biomed.Res 1996;29:162-173. [PubMed: 8812068]

De Luca M, Beckmann CF, De Stefano N, Matthews PM, Smith SM. fMRI resting state networks define distinct modes of long-distance interactions in the human brain. Neuroimage 2006;29:1359-1367. [PubMed: 16260155]

Detre JA, Alsop DC, Vives LR, Maccotta L, Teener JW, Raps EC. Noninvasive MRI evaluation of cerebral blood flow in cerebrovascular disease. Neurology 1998;50:633-641. [PubMed: 9521248]

Detre JA, Leigh JS, Williams DS, Koretsky AP. Perfusion imaging. Magn Reson.Med 1992;23:37-45. [PubMed: 1734182]

Fox MD, Corbetta M, Snyder AZ, Vincent JL, Raichle ME. Spontaneous neuronal activity distinguishes human dorsal and ventral attention systems. Proc.Natl.Acad.Sci.U.S.A 2006;103:10046-10051. [PubMed: 16788060]

Fox MD, Raichle ME. Spontaneous fluctuations in brain activity observed with functional magnetic resonance imaging. Nat.Rev.Neurosci 2007;8:700-711. [PubMed: 17704812]

Fox MD, Snyder AZ, Vincent JL, Corbetta M, Van Essen DC, Raichle ME. The human brain is intrinsically organized into dynamic, anticorrelated functional networks. Proc.Natl.Acad.Sci.U.S.A 2005;102:9673-9678. [PubMed: 15976020]

Fox MD, Zhang D, Snyder AZ, Raichle ME. The Global Signal and Observed Anticorrelated Resting State Brain Networks. J.Neurophysiol. 2009

Fransson P. Spontaneous low-frequency BOLD signal fluctuations: An fMRI investigation of the restingstate default mode of brain function hypothesis. Human Brain Mapping 2005;26:15-29. [PubMed: 15852468]

Gaa J, Warach S, Wen P, Thangaraj V, Wielopolski P, Edelman RR. Noninvasive perfusion imaging of human brain tumors with EPISTAR. Eur.Radiol 1996;6:518-522. [PubMed: 8798035]

Gong G, He Y, Concha L, Lebel C, Gross DW, Evans AC, Beaulieu C. Mapping Anatomical Connectivity Patterns of Human Cerebral Cortex Using In Vivo Diffusion Tensor Imaging Tractography. Cereb.Cortex. 2008

Greicius M. Resting-state functional connectivity in neuropsychiatric disorders. Curr.Opin.Neurol 2008;21:424-430. [PubMed: 18607202]

Greicius MD, Krasnow B, Reiss AL, Menon V. Functional connectivity in the resting brain: a network analysis of the default mode hypothesis. Proc.Natl.Acad.Sci.U.S.A 2003;100:253-258. [PubMed: 12506194]

Greicius MD, Srivastava G, Reiss AL, Menon V. Default-mode network activity distinguishes Alzheimer's disease from healthy aging: evidence from functional MRI. Proc.Natl.Acad.Sci.U.S.A 2004;101:4637-4642. [PubMed: 15070770]

Hagmann P, Cammoun L, Gigandet X, Meuli R, Honey CJ, Wedeen VJ, Sporns O. Mapping the structural core of human cerebral cortex. PLoS.Biol 2008;6:e159. [PubMed: 18597554]

He Y, Wang L, Zang Y, Tian L, Zhang X, Li K, Jiang T. Regional coherence changes in the early stages of Alzheimer's disease: a combined structural and resting-state functional MRI study. Neuroimage 2007;35:488-500. [PubMed: 17254803]

Hong LE, Gu H, Yang Y, Ross TJ, Salmeron BJ, Buchholz B, Thaker GK, Stein EA. Association of nicotine addiction and nicotine's actions with separate cingulate cortex functional circuits. Arch.Gen.Psychiatry 2009;66:431-441. [PubMed: 19349313]

Hudetz AG, Roman RJ, Harder DR. Spontaneous flow oscillations in the cerebral cortex during acute changes in mean arterial pressure. J.Cereb.Blood Flow Metab 1992;12:491-499. [PubMed: 1569142]

Iturria-Medina Y, Sotero RC, Canales-Rodriguez EJ, Aleman-Gomez Y, Melie-Garcia L. Studying the human brain anatomical network via diffusion-weighted MRI and Graph Theory. Neuroimage 2008;40:1064-1076. [PubMed: 18272400] 
Johnson NA, Jahng GH, Weiner MW, Miller BL, Chui HC, Jagust WJ, Gorno-Tempini ML, Schuff N. Pattern of cerebral hypoperfusion in Alzheimer disease and mild cognitive impairment measured with arterial spin-labeling MR imaging: initial experience. Radiology 2005;234:851-859. [PubMed: 15734937]

Jones SC, Williams JL, Shea M, Easley KA, Wei D. Cortical cerebral blood flow cycling: anesthesia and arterial blood pressure. Am.J.Physiol 1995;268:H569-H575. [PubMed: 7864181]

Kiviniemi V, Jauhiainen J, Tervonen O, Paakko E, Oikarinen J, Vainionpaa V, Rantala H, Biswal B. Slow vasomotor fluctuation in fMRI of anesthetized child brain. Magn Reson.Med 2000;44:373378. [PubMed: 10975887]

Liau J, Perthen JE, Liu TT. Caffeine reduces the activation extent and contrast-to-noise ratio of the functional cerebral blood flow response but not the BOLD response. Neuroimage 2008;42:296-305. [PubMed: 18514545]

Liu H, Liu Z, Liang M, Hao Y, Tan L, Kuang F, Yi Y, Xu L, Jiang T. Decreased regional homogeneity in schizophrenia: a resting state functional magnetic resonance imaging study. Neuroreport 2006;17:19-22. [PubMed: 16361943]

Liu TT, Wong EC. A signal processing model for arterial spin labeling functional MRI. Neuroimage 2005;24:207-215. [PubMed: 15588612]

Logothetis NK, Pauls J, Augath M, Trinath T, Oeltermann A. Neurophysiological investigation of the basis of the fMRI signal. Nature 2001;412:150-157. [PubMed: 11449264]

Long XY, Zuo XN, Kiviniemi V, Yang Y, Zou QH, Zhu CZ, Jiang TZ, Yang H, Gong QY, Wang L, Li KC, Xie S, Zang YF. Default mode network as revealed with multiple methods for resting-state functional MRI analysis. J.Neurosci.Methods 2008;171:349-355. [PubMed: 18486233]

Mazoyer B, Zago L, Mellet E, Bricogne S, Etard O, Houde O, Crivello F, Joliot M, Petit L, TzourioMazoyer N. Cortical networks for working memory and executive functions sustain the conscious resting state in man. Brain Research Bulletin 2001;54:287-298. [PubMed: 11287133]

Murphy K, Birn RM, Handwerker DA, Jones TB, Bandettini PA. The impact of global signal regression on resting state correlations: are anti-correlated networks introduced? Neuroimage 2009;44:893-905. [PubMed: 18976716]

Poline JB, Worsley KJ, Evans AC, Friston KJ. Combining spatial extent and peak intensity to test for activations in functional imaging. Neuroimage 1997;5:83-96. [PubMed: 9345540]

Raichle ME. Neuroscience. The brain's dark energy. Science 2006;314:1249-1250. [PubMed: 17124311]

Raichle ME, MacLeod AM, Snyder AZ, Powers WJ, Gusnard DA, Shulman GL. A default mode of brain function. Proc.Natl.Acad.Sci.U.S.A 2001;98:676-682. [PubMed: 11209064]

Shulman GL, Fiez JA, Corbetta M, Buckner RL, Miezin FM, Raichle ME, Petersen SE. Common blood flow changes across visual tasks .2. Decreases in cerebral cortex. Journal of Cognitive Neuroscience 1997;9:648-663.

Stevens MC, Pearlson GD, Calhoun VD. Changes in the interaction of resting-state neural networks from adolescence to adulthood. Hum.Brain.Mapp. 2009

Strouse JJ, Cox CS, Melhem ER, Lu H, Kraut MA, Razumovsky A, Yohay K, van Zijl PC, Casella JF. Inverse correlation between cerebral blood flow measured by continuous arterial spin-labeling (CASL) MRI and neurocognitive function in children with sickle cell anemia (SCA). Blood 2006;108:379-381. [PubMed: 16537809]

van den Heuvel MP, Stam CJ, Boersma M, Hulshoff Pol HE. Small-world and scale-free organization of voxel-based resting-state functional connectivity in the human brain. Neuroimage 2008;43:528539. [PubMed: 18786642]

Welsh RC, Chen AC, Taylor SF. Low-Frequency BOLD Fluctuations Demonstrate Altered Thalamocortical Connectivity in Schizophrenia. Schizophr.Bull. 2008

Wong EC, Buxton RB, Frank LR. Quantitative imaging of perfusion using a single subtraction (QUIPSS and QUIPSS II). Magn Reson.Med 1998;39:702-708. [PubMed: 9581600]

Wu CW, Gu H, Lu H, Stein EA, Chen JH, Yang Y. Mapping functional connectivity based on synchronized CMRO2 fluctuations during the resting state. Neuroimage 2009;45:694-701. [PubMed: 19280693]

Wu T, Long X, Zang Y, Wang L, Hallett M, Li K, Chan P. Regional homogeneity changes in patients with Parkinson's disease. Hum.Brain Mapp 2009;30:1502-1510. [PubMed: 18649351] 
Wu T, Zang Y, Wang L, Long X, Li K, Chan P. Normal aging decreases regional homogeneity of the motor areas in the resting state. Neurosci.Lett 2007;423:189-193. [PubMed: 17709202]

Yang Y, Glover GH, van Gelderen P, Patel AC, Mattay VS, Frank JA, Duyn JH. A comparison of fast MR scan techniques for cerebral activation studies at 1.5 tesla. Magn Reson.Med 1998;39:61-67. [PubMed: 9438438]

Ye FQ, Berman KF, Ellmore T, Esposito G, van Horn JD, Yang Y, Duyn J, Smith AM, Frank JA, Weinberger DR, McLaughlin AC. H(2)(15)O PET validation of steady-state arterial spin tagging cerebral blood flow measurements in humans. Magn Reson.Med 2000;44:450-456. [PubMed: 10975898]

Zang Y, Jiang T, Lu Y, He Y, Tian L. Regional homogeneity approach to fMRI data analysis. Neuroimage 2004;22:394-400. [PubMed: 15110032]

Zang YF, He Y, Zhu CZ, Cao QJ, Sui MQ, Liang M, Tian LX, Jiang TZ, Wang YF. Altered baseline brain activity in children with ADHD revealed by resting-state functional MRI. Brain Dev 2007;29:83-91. [PubMed: 16919409]

Zhu CZ, Zang YF, Cao QJ, Yan CG, He Y, Jiang TZ, Sui MQ, Wang YF. Fisher discriminative analysis of resting-state brain function for attention-deficit/hyperactivity disorder. Neuroimage 2008;40:110 120. [PubMed: 18191584]

Zou QH, Zhu CZ, Yang Y, Zuo XN, Long XY, Cao QJ, Wang YF, Zang YF. An improved approach to detection of amplitude of low-frequency fluctuation (ALFF) for resting-state fMRI: fractional ALFF. J.Neurosci.Methods 2008;172:137-141. [PubMed: 18501969]

Zuo, X.; Di Martino, A.; Kelly, C.; Shezhad, ZE.; Gee, D.; Klein, DF.; Castellanos, FX.; Biswal, BB.; Milham, MP. 15th Annual Meeting of Organization for Human Brain Mapping, poster 548.; 2009. 

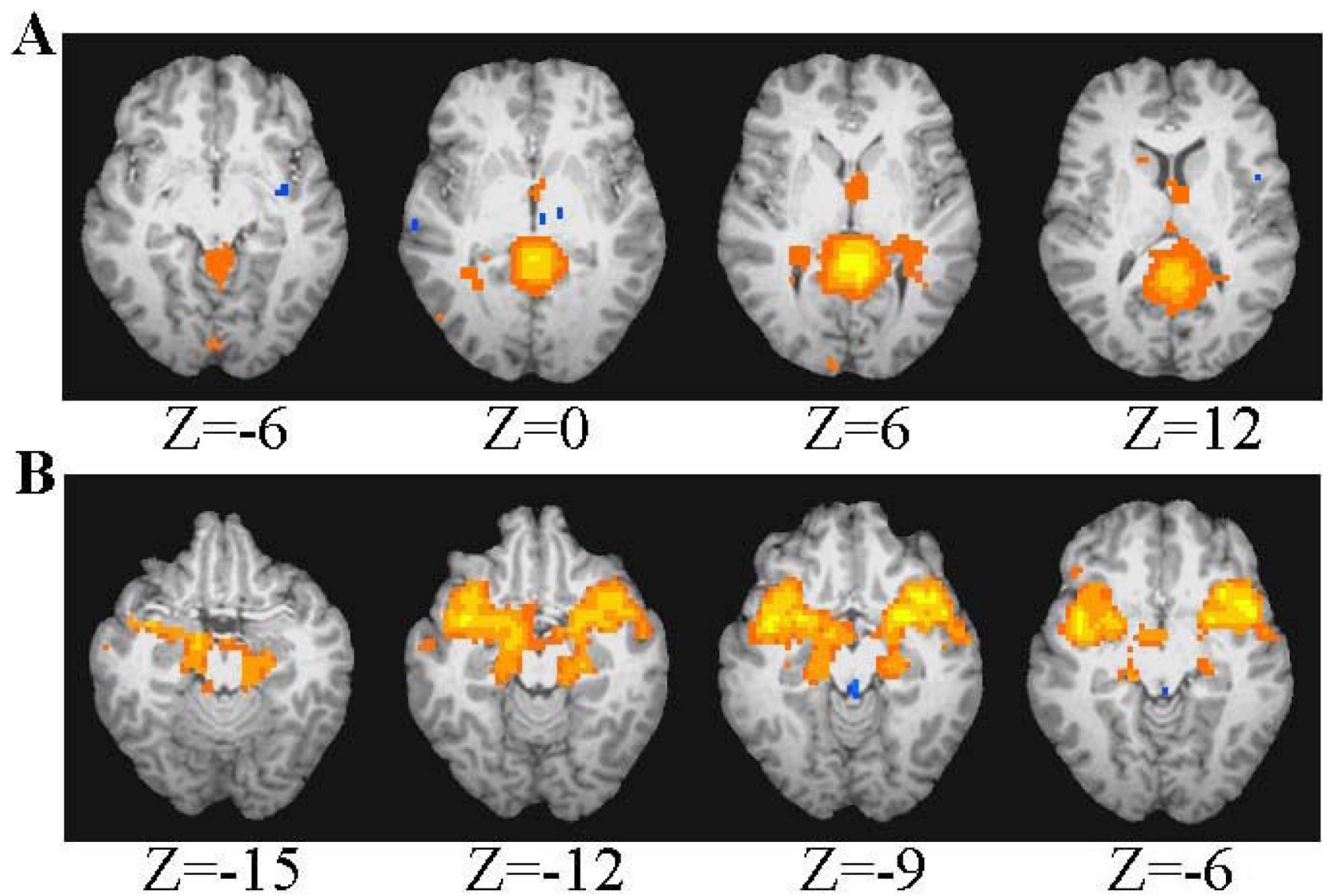

Fig. 1.

Spatial patterns of two ICA components that were related to physiological noise estimated from the group of 12 subjects. The numbers below the images refer to the $\mathrm{z}$ coordinates in Talairach space. A. cerebrospinal fluid. B. blood vessels. 


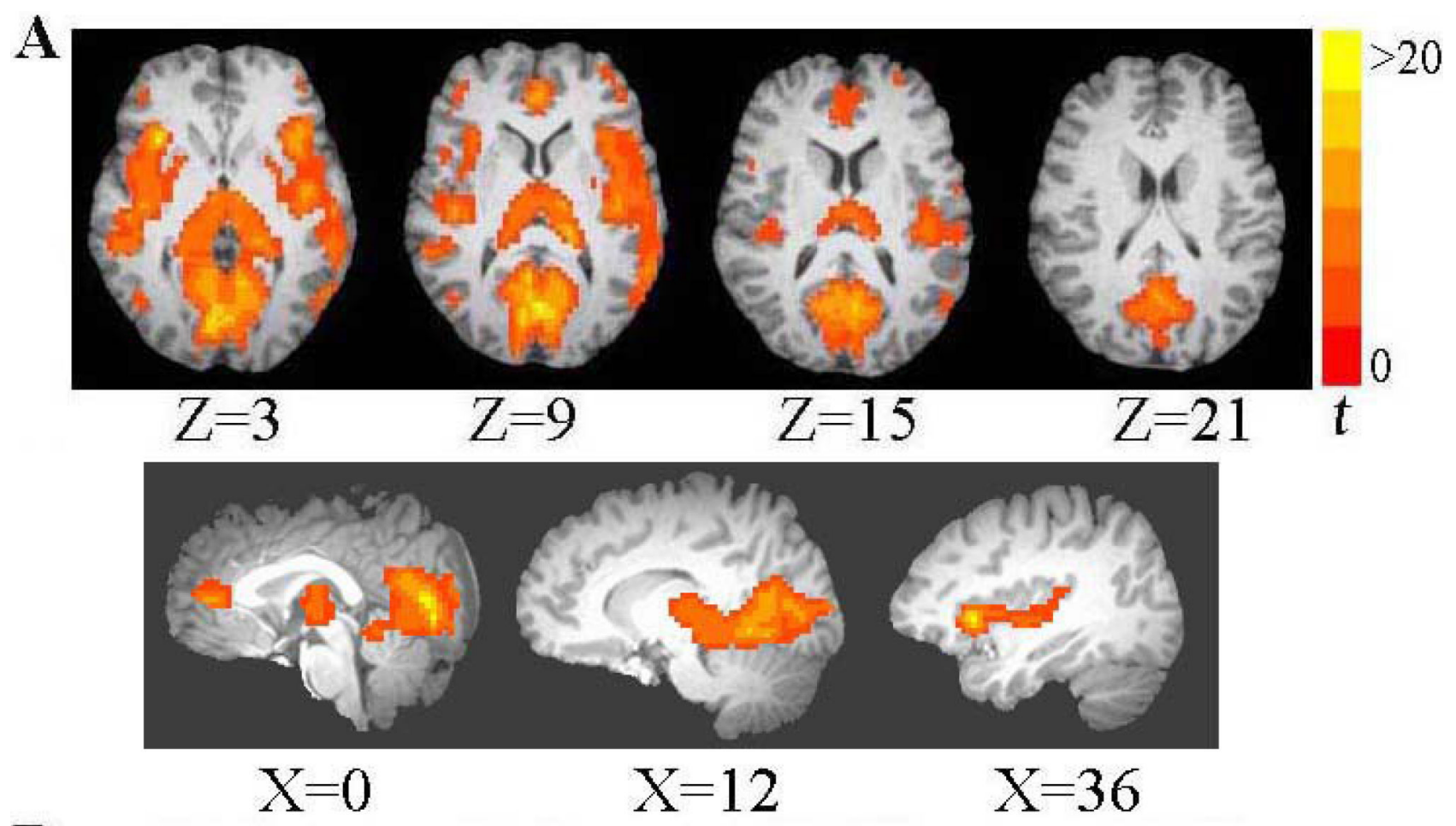

B

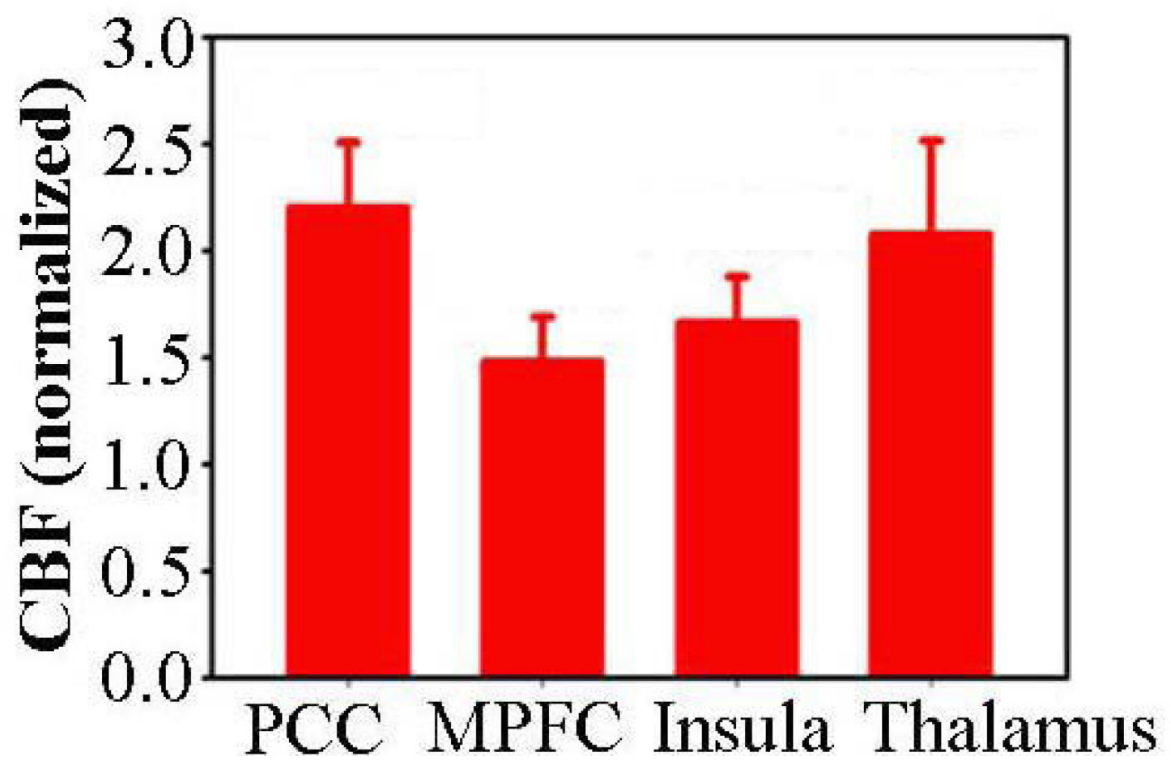

Fig. 2.

Static characteristics of the CBF signals from the group of 12 subjects. A. The numbers below the images refer to the $\mathrm{z}$ coordinates (axial view) or $\mathrm{x}$ coordinates (sagittal view) in Talairach space. B. Static CBF in the PCC $(t=13.60)$, MPFC $(t=7.98)$, insula/STG $(t=10.58)$ and thalamus $(t=8.49)$ were significantly higher than the whole brain average (i.e., 1). Data plotted are mean + std. 
A

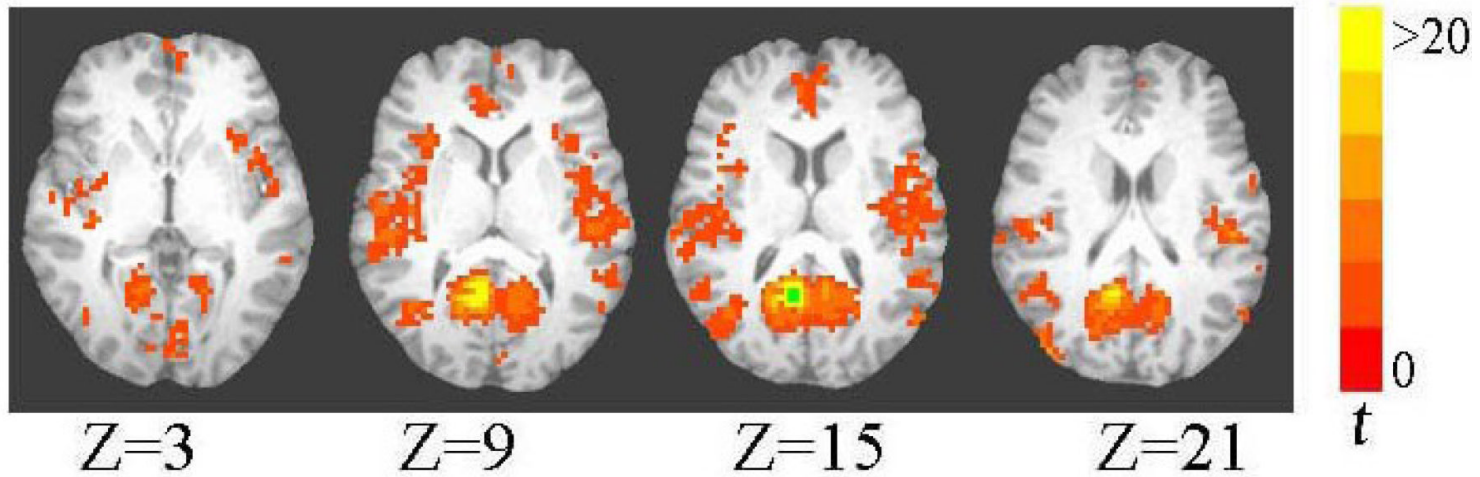

B
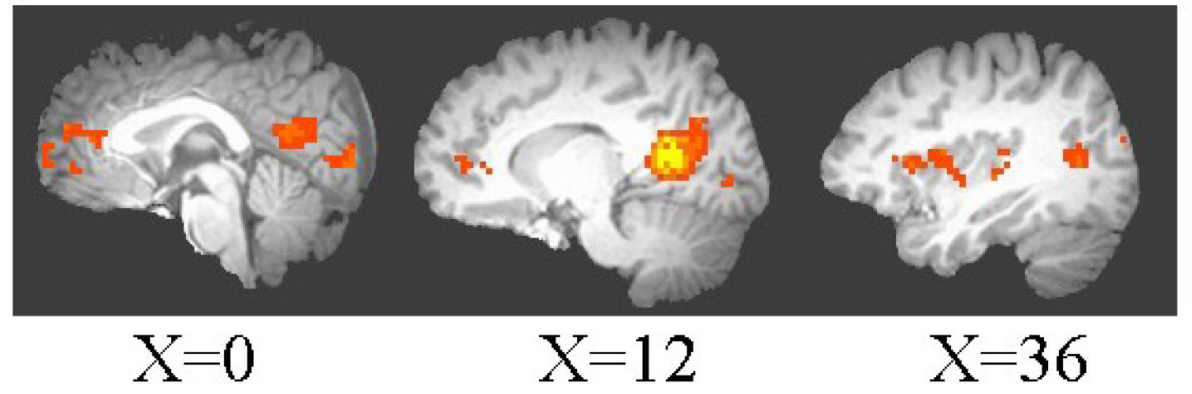

$\mathrm{X}=36$

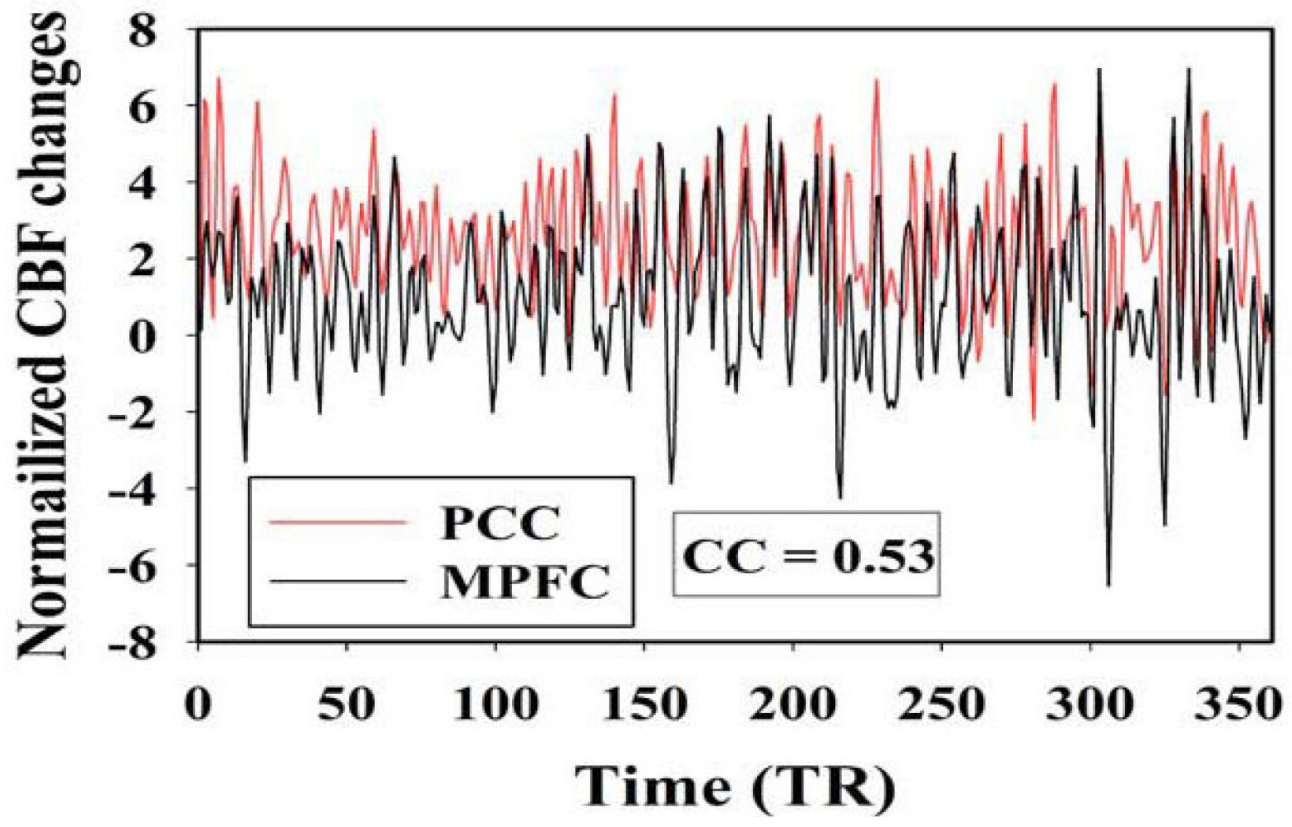

Fig. 3A.

Functional connectivity between the CBF changes in the PCC and other brain regions from the group of 12 subjects. The numbers below the images refer to the $\mathrm{z}$ coordinates (axial view) or $\mathrm{x}$ coordinates (sagittal view) in Talairach space. The green square indicates the location of the PCC seed. B. Normalized time courses retrieved from the PCC and MPFC from a representative subject. 

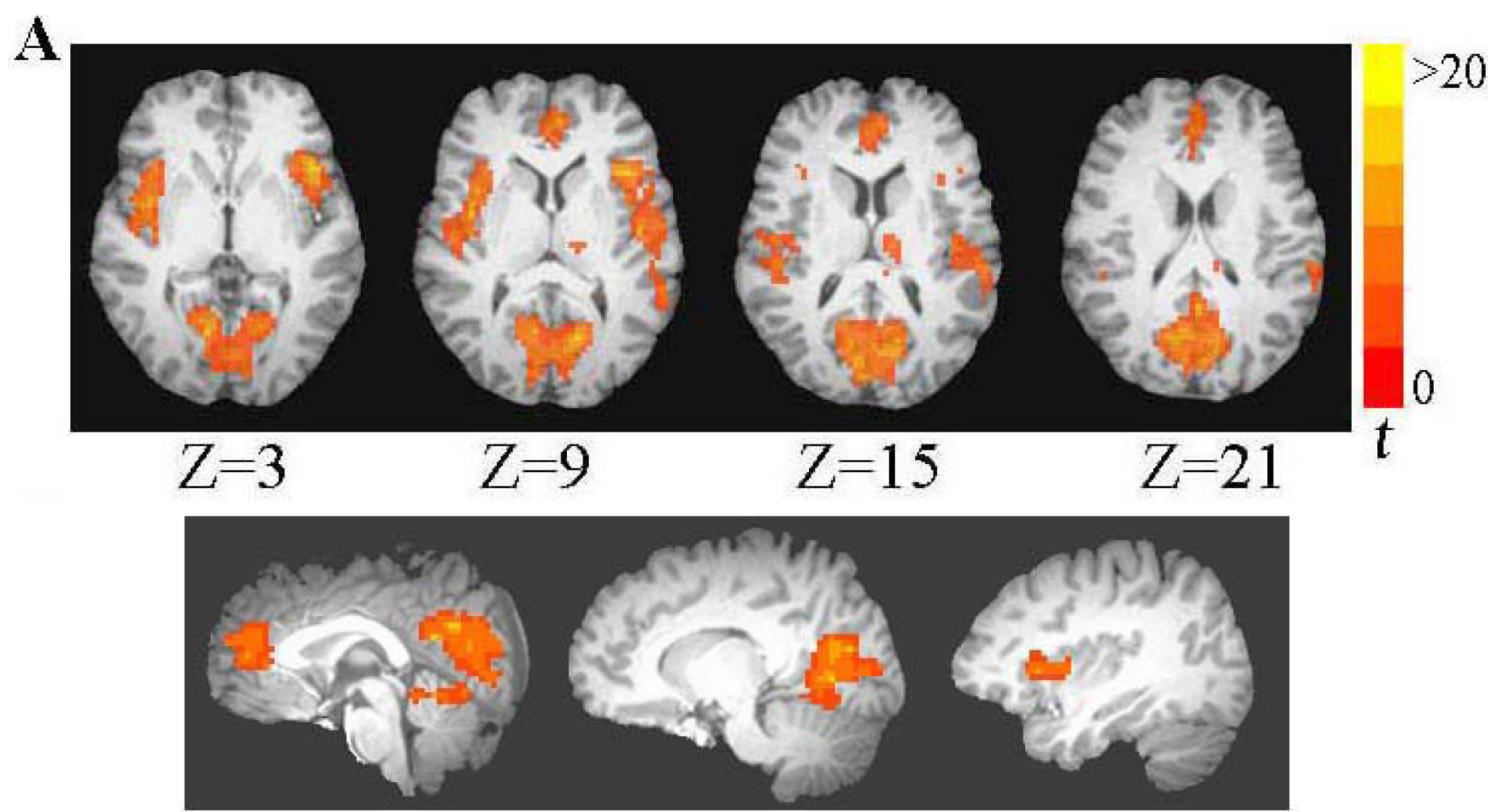

B
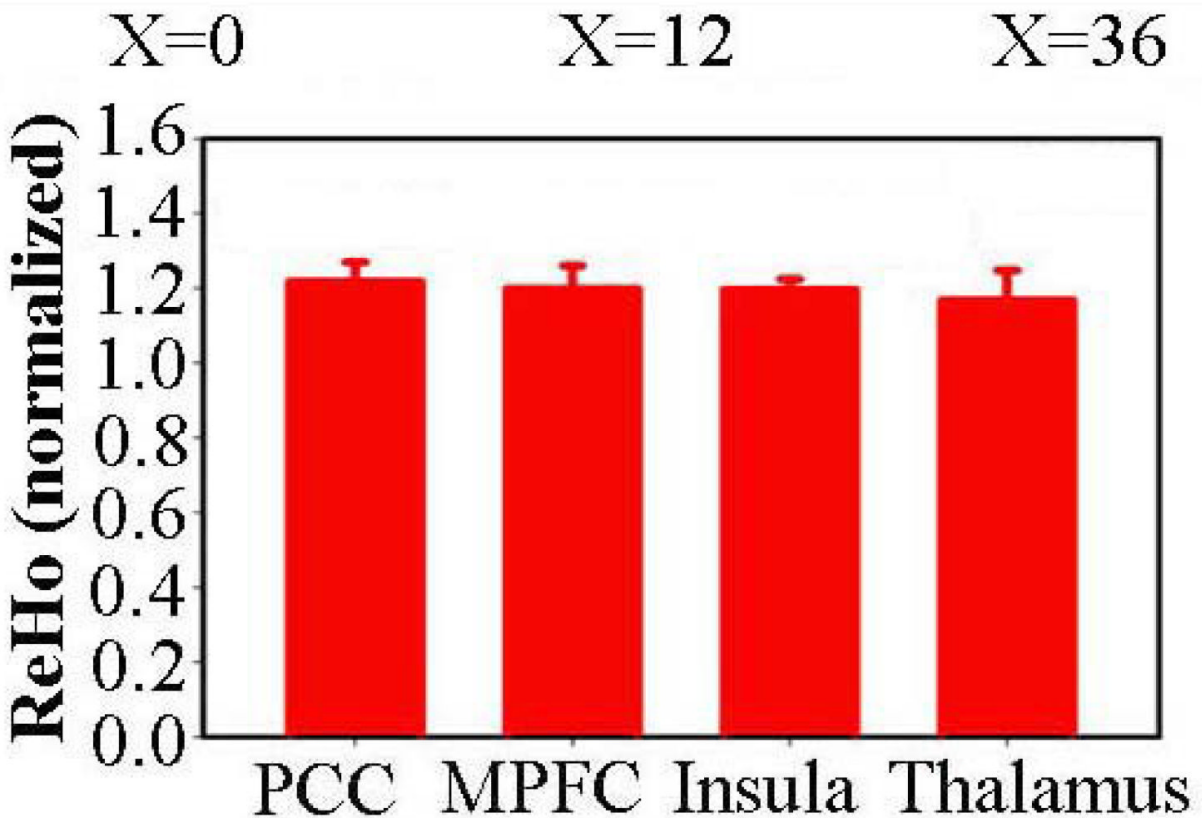

Fig. 4.

ReHo results of dynamic CBF signals from the group of 12 subjects. A. The numbers below the images refer to the $\mathrm{z}$ coordinates (axial view) or $\mathrm{x}$ coordinates (sagittal view) in Talairach space. B. ReHo values in the PCC ( $t=14.03)$, MPFC $(t=10.79)$, insula/STG $(t=22.80)$ and thalamus $(t=7.08)$ were significantly higher than the whole brain average (i.e., 1). Data plotted are mean + std. 


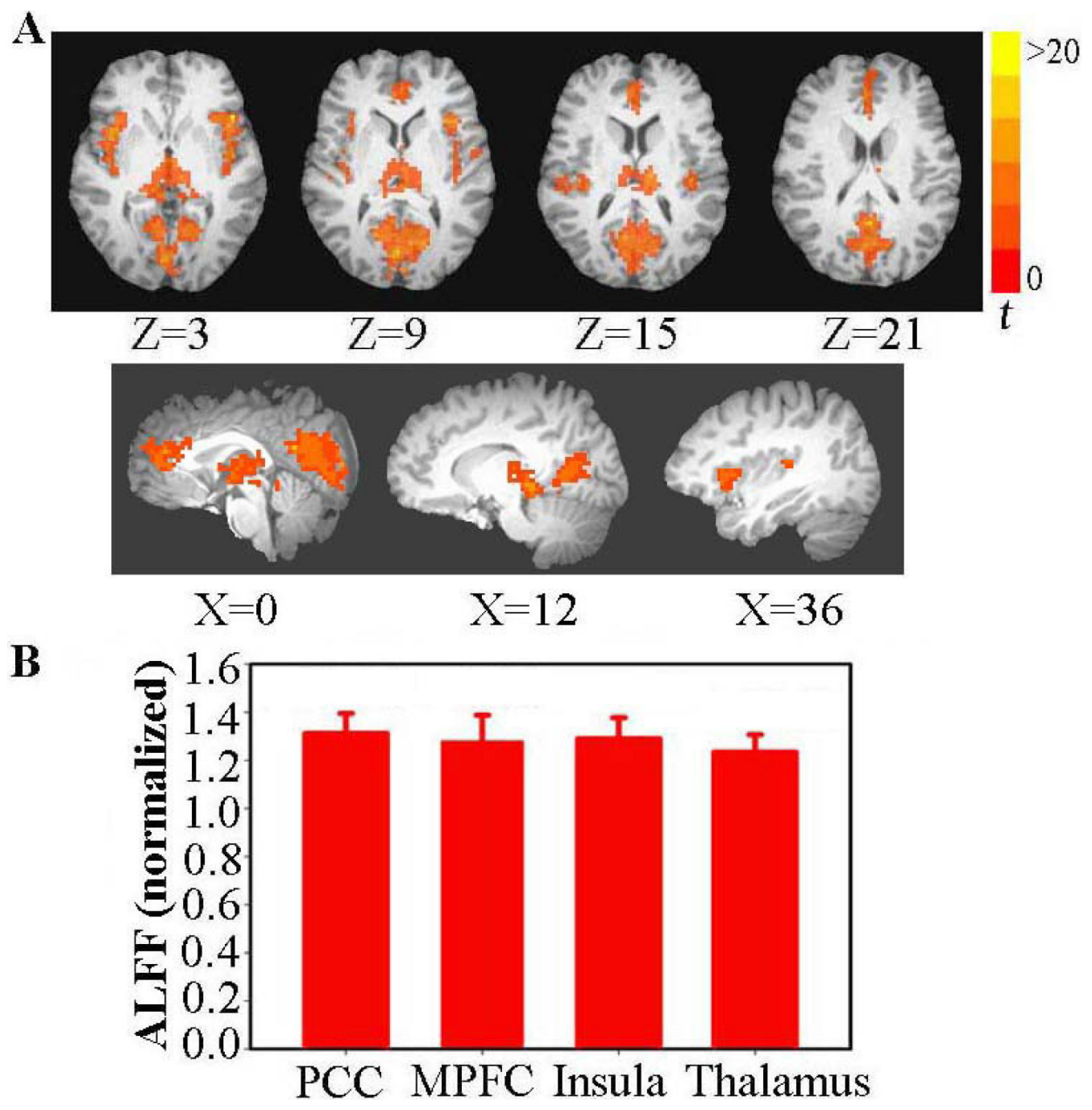

Fig. 5.

ALFF results of dynamic CBF signals from the group of 12 subjects. A. The numbers below the images refer to the $\mathrm{z}$ coordinates (axial view) or $\mathrm{x}$ coordinates (sagittal view) in Talairach space. B. ALFF values within the PCC $(t=12.98)$, MPFC ( $t=9.08)$, insula/STG $(t=11.43)$ and thalamus $(t=11.09)$ were significantly higher than the whole brain average (i.e., 1$)$. Data plotted are mean + std. 


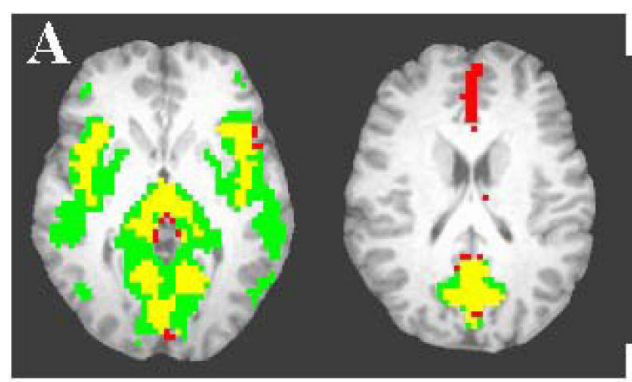

$Z=3$

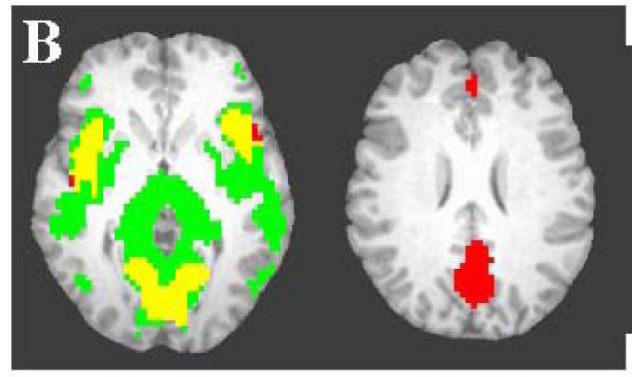

$Z=3$
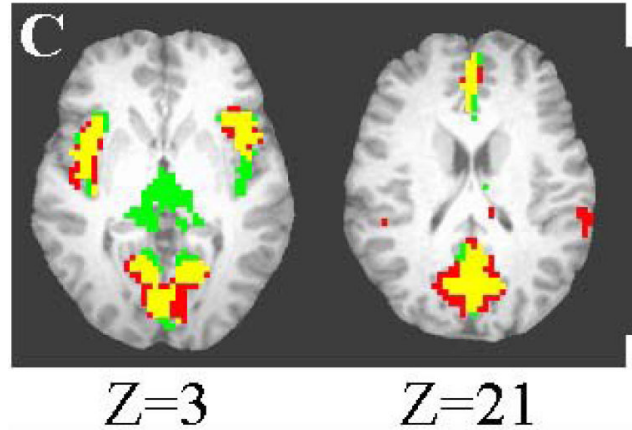

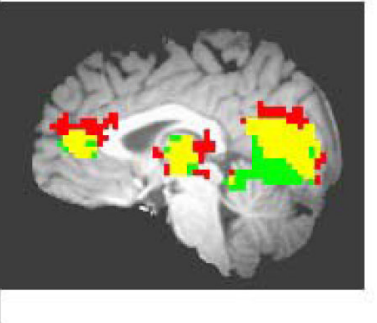

$X=-2$

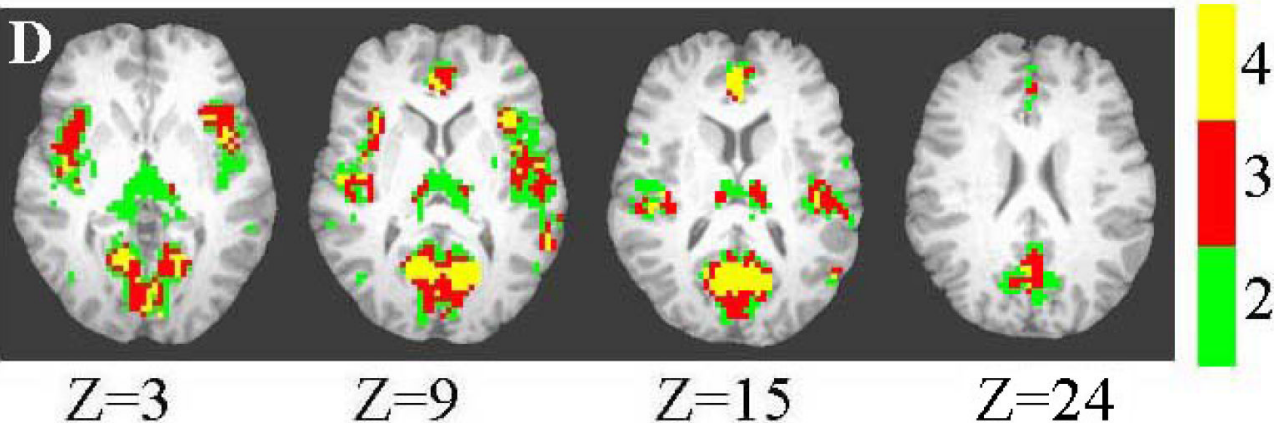

Fig. 6.

Spatial overlap of static and dynamic characteristics. The numbers below the images refer to the $\mathrm{z}$ coordinates (axial view) or $\mathrm{x}$ coordinates (sagittal view) in Talairach space. A. Overlap of static CBF and ALFF maps. Regions in yellow showed significance in both static CBF (in green) and ALFF (in red) maps. B. Overlap of static CBF and ReHo maps. Regions in yellow showed significance in both static CBF (in green) and ReHo (in red) maps. C. Overlap of ALFF and ReHo maps. Regions in yellow showed significance in both ALFF (in green) and ReHo (in red) maps. D. Overlap of $t$-maps of static CBF, PCC-based correlation, ALFF and ReHo. Regions in yellow, red and green indicate that these regions showed significant results in all four, three, and two of $t$-maps, respectively. 